\title{
Managing a Peritoneal Dialysis Patient with High Risk for Cardiovascular Disease
}

\author{
Roberto Pecoits-Filho \\ Pontifícia Universidade Católica do Paraná, Curitiba, Brazil \\ Coordinator: Konniev Rodriguez, President of the Colombian Congress of Nephrology, 2009
}

\section{Presenter: Roberto Pecoits-Filho, MD, PhD}

Presented during the Colombian Congress of Nephrology, 2009

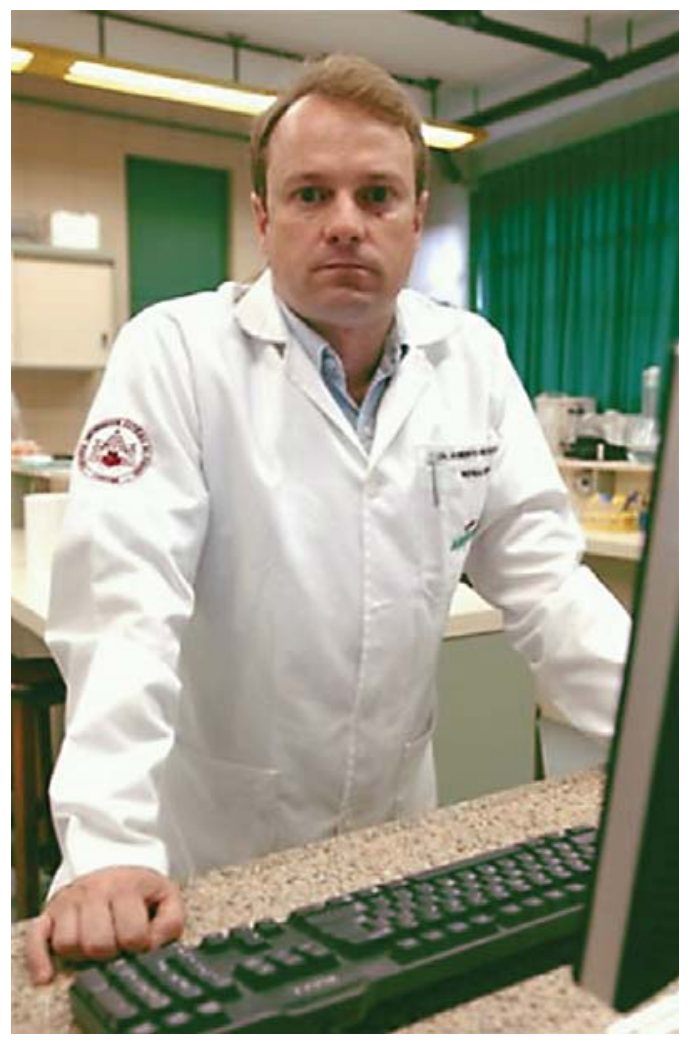

Roberto Pecoits-Filho is Professor of Medicine at the Pontifícia Universidade Católica do Paraná and Director of the PhD and Master Programs in Health Sciences at the same university. Dr. Pecoits-Filho received his MD in 1993, trained in Internal Medicine and Nephrology in Curitiba and was a fellow at the University of Missouri. He received a PhD from the University of São Paulo and was a visiting scholar at the Karolinska Institute.

\section{Case Presentation}

A patient presented to the clinic with a chief complaint of shortness of breath. The patient was 56 years old from Curitiba, Brazil, worked as a bus driver, and was married with two children. They lived in a simple house in the suburbs and he was the only working family member. He studied until the first grade and the family income was the equivalent of USD 700. He started to become overweight at the age of 30 , and felt well until he was diagnosed with diabetes after a hospital admission to investigate blurred vision at the age of 42 . He was $161 \mathrm{~cm}$ tall and weighed 60 $\mathrm{kg}$. Glycemic control was very difficult from the beginning, mainly due to the fact that the patient did not follow dietary guidelines or take his prescribed medication. Hypertension developed 6 years after the diagnosis of diabetes, which proved to be difficult to control despite the prescription of different drugs. At the age of 54 , he was referred to the renal clinic as he presented with generalized edema.

\section{KARGER}

Fax +41613061234 E-Mail karger@karger.ch www.karger.com
(C) 2010 S. Karger AG, Basel

$1660-2110 / 10 / 1164-0283 \$ 26.00 / 0$

Accessible online at: www.karger.com/nec
Roberto Pecoits-Filho

Pontifícia Universidade Católica do Paraná

Av. Imaculada Conceição, 1155

Curitiba, PR 80215-901 (Brazil)

Tel. +55 4132712 285, Fax +55 4132711 657, E-Mail r.pecoits@ pucpr.br 
At that moment, he presented an elevation in plasma creatinine $(2.7 \mathrm{mg} / \mathrm{dl}-\mathrm{eGFR} 29 \mathrm{ml} / \mathrm{min})$ and $2.4 \mathrm{~g}$ of proteinuria. Over the following 2 years, his GFR progressively fell until he presented to the renal clinic with nausea, weight loss, generalized edema and an eGFR of $13 \mathrm{ml} / \mathrm{min}$. Blood glucose was $70 \mathrm{mg} \%$, Hb $9.5 \mathrm{~g} \%$, K $4.5 \mathrm{mEq} / \mathrm{l}$, glucose $160 \mathrm{mg} \%$, creatinine $4.7 \mathrm{mg} \%$, P $5.7 \mathrm{mg} \%$, LDL $90 \mathrm{mg} \%$, TG $100 \mathrm{mg} \%$ and PTH $80 \mathrm{pg} / \mathrm{ml}$. Serum albumin was $2.8 \mathrm{mg} \%$. His prescription at that time was based on enalapril 20 $\mathrm{mg}$ /day, hydrochlorothiazide $25 \mathrm{mg}$ /day and insulin $10 \mathrm{U} / 5 \mathrm{U}$. After suspending the ACEi and insulin, and failing to compensate the patient, his nephrologist decided to initiate dialysis treatment, choosing peritoneal dialysis (PD) after talking to the patient and his family about the analysis of the options.

The patient and his family were very much against hemodialysis (HD) due to a bad family experience and the fact that the patient lived $80 \mathrm{~km}$ from the nearest HD center. A peritoneal catheter was implanted by a nephrologist and the patient was initiated on $4 \times 1.5 \% 2$-liter exchanges per day. After 30 days, he presented to the clinic with edema and shortness of breath, his body weight increased to $75 \mathrm{~kg}$ and his blood pressure increased to 160/100 mm Hg. Lab exams presented at the evaluation showed: Hb $8.9 \mathrm{~g} \%$, K $4.5 \mathrm{mEq} /$, glucose $160 \mathrm{mg} \%$, creatinine $4.7 \mathrm{mg} \%, \mathrm{P}$ $5.7 \mathrm{mg} \%$, LDL $130 \mathrm{mg} \%$, TG $500 \mathrm{mg} \%$ and PTH $80 \mathrm{pg} / \mathrm{ml}$. Serum albumin was $2.8 \mathrm{mg} \%$. The patient was on nifedipine (40 mg/day) and calcium carbonate ( $2 \mathrm{~g} /$ day). An X-ray showed that the catheter was in a good position. His urine output was $1,300 \mathrm{ml}$ and total drained volume during $24 \mathrm{~h}$ on the baseline prescription was 9 liters. Ultrafiltration after $4 \mathrm{~h}$ of a $4.25 \%$ exchange resulted in $2,250 \mathrm{ml}$. A peritoneal equilibration test was performed, resulting in a $\mathrm{D} / \mathrm{P}$ of creatinine at $4 \mathrm{~h}$ of 0.81 . His weekly peritoneal $\mathrm{Kt} / \mathrm{V}$ with the original prescription was 1.6.

\section{Discussion}

The prevalence of chronic kidney disease (CKD) is increasing worldwide and a growing number of complicated patients are starting renal replacement therapy. It appears that this problem is particularly challenging in developing countries. According to the Latin American Society of Nephrology and Hypertension (SLANH) registry, a prevalence of dialysis (both HD and PD) and renal transplant of 363 patients per 1,000,000 population was reported, $30 \%$ of diabetics [1]. In the same document, an acceptance rate of 103 new patients per 1,000,000 population for renal replacement therapy was observed, even though this is highly heterogeneous in the region [1]. In Latin America, HD is the predominant form of end-stage renal disease (ESRD) replacement therapy, although four countries (Colombia, Guatemala, El Salvador and Mexico) employ PD in $30 \%$ or more of the ESRD cases [2]. In particular, Mexico is the largest user of PD in the world, and it is estimated that approximately $25 \%$ of the world's PD population comes from Latin America.
In Colombia, PD has a large penetration (over 40\%), and currently $34 \%$ of PD patients utilize cyclers. In Colombia, there is one nephrologist for every 80 dialysis patients and the ratio of nurse/patient in the PD program is $1 / 45$. Nephrologists are currently implanting catheters in half of the patients. Gross mortality in PD is $16 \%$, slightly lower than the HD mortality rate (19\%). The peritonitis rate in automated PD (APD) is 1 episode/32 patient/ month; for continuous ambulatory PD (CAPD) the peritonitis rate is 1 episode/26 patient/month. Staphilococcus epidermidis is the main microorganism causing peritonitis. The average volume prescribed is 7.6 liters for CAPD and 11.2 liters for APD. The controllable PD drop-out (transfer to HD only) is 16\% per year. The government covers reimbursement, and the value paid is nearly the same for HD and PD [2].

\section{Was PD a Good Modality Option for This Patient?}

Deciding which is the best renal replacement therapy for each patient in a particular moment is a difficult task. However, it is an important decision since many CKD patients will need to change modality due to limitations of the methods available. To be a first-line option, transplant needs time for follow-up during the pre-dialytic care and an absence of contraindications for surgery or immunosupression. Unfortunately, most patients starting dialysis in developing countries, such as in Latin America, at the present time have a short time of followup or have comorbidities that limit the access to transplantation, leaving dialysis, namely HD or PD, as the only form of renal replacement therapy [2].

If we search for the randomized controlled trials performed to solve the issue of dialysis modality selection based on the knowledge of the therapy that provides the best survival and quality of life, no definitive studies guide the evidence. In fact, there was only one single attempt to perform a randomized controlled study comparing $\mathrm{HD}$ to $\mathrm{PD}$, which was terminated due to the very low number of patients who allowed themselves to be randomized [3]. Therefore, when selecting the best therapy for each patient, we should analyze observational data from registries. In summary, studies that looked at differences in specific regions found lower mortality for PD patients during the first 1-2 years, an advantage that disappears after approximately 2 years of follow-up [4]. A recent observational study performed in Colombia, which had a proportional distribution of patients on PD and HD, showed equivalent survival for the two groups, despite the fact that PD patients presented more comorbidities and less access to medical care [5]. 
Another important aspect of modality selection is to carefully search for contraindications and particular groups that may benefit from a particular modality. Absolute contraindications for PD or HD are rare and particularly younger and non-diabetic patients appear to present a superior survival on PD [6]. Particularly in countries with limited resources, the total cost of therapy to the public system should be analyzed [7]. Moreover, demographic and socioeconomic factors should also be analyzed in the dialysis modality decision.

This patient lives far away from the center, which favors PD. On the other hand, his socioeconomic condition traditionally has been considered a limitation. Although poor family income may sometimes be considered a contraindication for PD, no consistent data links economical status to clinical outcome in this group of patients. Recently, however, a large Brazilian cohort study showed no impact of poverty on outcome [8]. In total, 1,952 incident PD patients were enrolled in this prospective cohort, studied according to monthly family income (Brazilian minimum wage (MW): USD 4.94/day) and divided into three groups: low $(<2 \mathrm{MW})$, middle and high economic status ( $>5 \mathrm{MW}$ ). The follow-up period ranged from 3 to 34 months, and during this period 307 patients died. $\mathrm{Cu}-$ mulative patient and technique survival did not vary significantly among groups. In a multivariate Cox regression analysis, no statistical difference in patient survival was observed. This report may change the policies in terms of access to PD for patients with a low socioeconomic level.

PD adapted in terms of patient education or home infrastructure to poor and illiterate patients may be a viable option to optimize the access to renal replacement therapies in countries with low resources. Therefore, looking at this patient's clinical and demographic conditions, it seems fair to consider both renal replacement therapies as equivalent in terms of providing the best clinical outcome. Patient and family opinion should drive the choice of dialysis modality.

\section{Why Did the Patient Present Fluid Overload So Early in the Course of the Treatment?}

The investigation of fluid overload in this patient showed that the cause for the low drainage volume was most likely due to limitation in the capacity to generate enough ultrafiltration to keep a normal volemic status. Indeed, the combination of tests $(<400 \mathrm{ml}$ generated with a hypertonic solution 4 -hour dwell and a high $\mathrm{D} / \mathrm{P}$ of creatinine ratio) suggested that the patient presented ultrafiltration failure. The interpretation of this evaluation did not allow for an analysis of a specific cause since aquaporin failure, increase in lymphatic reabsorption or an increase in the peritoneal vascular area could have had a similar clinical presentation. However, a large peritoneal surface and increased intrinsic vascular permeability are by far the most common mechanisms behind ultrafiltration loss. Although it is very common in patients on longterm $\mathrm{PD}$, it is possible that a fast peritoneal transport rate occurs at the early stages of PD treatment. In these cases, peritoneal alterations appear as a consequence of the impact of comorbidities on the peritoneum. Since the patient was a diabetic and had significant comorbidity, it is likely that he already presented peritoneal structural and functional alterations at the start of PD treatment [9]. With the decrease in ultrafiltration as a consequence of the fast glucose absorption rate over the dwell period, the patient will be prone to fluid overload, particularly if low compliance to a low sodium diet and dialysis prescription accompanies this condition.

What Would Be the Changes in the Dialysis and Medication Prescription in Order to Decrease Mortality and Morbidity in This Patient?

The clinical case presented represents an increasingly common and challenging situation. For many years, due to the difficulty in generating knowledge in the field of risk factors to mortality disease in $\mathrm{CKD}$, managing a high-risk patient on dialysis, particularly on PD, was a frustrating task. Currently, many traditional and nontraditional risk factors have been identified, and interventions to extend patient survival are becoming feasible and effective. In the following section, I will discuss the most important risk factors and their recommended management. Most likely, these multifactorial actions, including optimized PD prescription (with the goal of achieving effective solute and fluid removal with minimal glucose exposure) will provide this patient with improvement in clinical outcome.

Fluid Overload and Hypertension

$\mathrm{PD}$ is associated with difficulties in maintaining normal fluid status, particularly after patients become anuric, due to its limitations in ultrafiltration. Fluid overload, with or without hypertension, is also more frequent in fast transporters [10]. This may be due to multiple reasons. First, there is a tradition in many clinics to tell patients in the pre-dialysis care that they do not need the same restriction of salt or liquid as HD patients. This concept has changed and may have an important impact on patient outcome if salt and water restriction is not equal- 
ly oriented to HD and PD patients. Salt restriction must be reinforced to achieve an even lower sodium intake in patients with signs of fluid overload. Monitoring patient compliance to the dietary orientation as well as compliance to the dialysis prescription is very important. Utilization of loop diuretics, even in large doses, increases the urine volume effectively and should be used as a tool to increase fluid removal [11] without the need for increasing the tonicity of $\mathrm{PD}$ solutions, which are associated with peritoneal membrane alterations and systemic metabolic complications largely due to high concentrations of glucose in the PD fluid. Nevertheless, strategies to increase ultrafiltration, such as APD, shorter CAPD dwells and the use of icodextrin solutions, have been associated with improvements in technique and patient survival [12]. In conclusion, a combination of nurse (education, monitoring compliance), dietitian (dietary counseling and fluid status monitoring) and physician (diuretics, dialysis prescription) is essential for achieving success in controlling this important comorbidity in PD patients. Correcting the disturbances of fluid status in PD patients with low ultrafiltration may reverse the high mortality risk observed in patients with a fast peritoneal transport rate.

\section{Glucose Metabolism/Lipids}

Patients on long-term PD present with a unique metabolic disturbance related to uremic toxicity and glucose absorption. These complex metabolic disturbances in glucose/lipid metabolism are extremely important among these risk factors as they permeate all stages of the kidney-cardiovascular interaction, from the development of diabetic nephropathy through the genesis of uremic insulin resistance to body composition changes [13]. In addition to the disturbances of glucose and lipid metabolism observed in CKD, PD-related risk factors (mainly induced by high glucose exposure) characterize a complex metabolic disorder that potentially harms the patient. The prolonged use of PD solutions with a high glucose concentration generates a series of consequences not only in the peritoneal membrane, but also at the systemic level, which vary from hyperglycemia and insulin resistance to dyslipidemia and central obesity. These metabolic abnormalities are common findings in PD patients and may directly influence patient outcome. Lifestyle and dietary modifications, as well as pharmacological interventions, may be particularly important in minimizing these risk factors and particularly for avoiding unnecessary use of hypertonic solutions. Glucose-sparing solutions may represent an additional strategy to change the PD-induced metabolic profile.
Anemia

Anemia is a common complication of CKD associated with adverse cardiovascular complications and is an independent risk factor for poor outcomes in patients with CKD in all stages, particularly in patients requiring renal replacement therapy [14]. Specific mechanisms leading to renal anemia in CKD have been investigated, and currently iron deficiency and a decrease in erythropoietin production or resistance to its actions are clearly central factors in this important complication of CKD. Moreover, efficient and safe treatment of anemia is largely available, large and well-designed trials have been finalized and evidence-based guidelines on how to treat renal anemia have been developed. Treatment of anemia has had an important impact on CKD patients' quality of life and clinical outcome.

$\mathrm{HD}$ and PD present striking differences that may affect anemia prevalence, diagnosis and control. Despite the fact that PD is considered an equivalent alternative to HD and is utilized universally, most of the data supporting anemia prevalence, associated factors and clinical guidelines for treatment originate from the studies based in the HD population. Studies focusing on anemia in $\mathrm{PD}$ patients need to be performed to guide clinical practice.

\section{Conclusions}

Obviously, managing a patient with so many risk factors for early mortality involves even more targets, including mineral metabolism and perhaps unknown risk factors related to uremia itself. Also, a large variety of cardioprotective drugs are currently being tested in large international clinical trials, and their results may introduce new therapeutic tools to those described. The results of this are that these advances in the understanding of risk factors, assessment of patients, and evolution in the PD technique and solutions will greatly affect the way we manage patients at high risk, expanding the role of PD in renal replacement therapy.

\section{Questions and Answers}

Leonardo Marin, Nephrologist from the Fundacion Leonor Goeckel, Cali, Colombia: This is a case of a patient that we see quite frequently: a diabetic man, with CKD and a low socioeconomic level. He arrives late to renal care with a low GFR and uremic symptoms. He is short 
and weighs only $65 \mathrm{~kg}$. It does not seem that he has any absolute contraindication for $\mathrm{PD}$, he seems willing to do self-care and lives far from a dialysis center. What calls my attention in this case is the lack of information regarding how training was performed. He has UF failure and a fast peritoneal transport. Certainly, this patient would not react well in a long dwell with $1.5 \%$ solutions, and the problem may have started with the choice of low glucose concentration. When the patient is admitted with edema, diuretics should be prioritized, and good glycemic control using high glucose concentration could minimize the renal function deterioration. Residual renal function should be always preserved. Although compliance could be an additional problem, I believe that in this case the choice of glucose concentration is the central problem. If available, the use of icodextrin-based solutions could be a very interesting alternative.

Dr. Pecoits-Filho: Just an additional comment on the preservation of renal function with renoprotective drugs, which is very important. Perhaps at the late stages of $\mathrm{CKD}$, there is a limitation for the use of ACEi or ARB due to the risk of hyperkalemia in some patients, but after the initiation of PD, these drugs are safe to use and efficient in slowing the progression of residual renal dysfunction in PD patients, which was confirmed by two randomized controlled trials.

German Munoz, Nephrologist from Cucuta, Colombia: In relation to Dr. Marin's comment about the case, I agree on achieving ultrafiltration, keeping the monitorization and control of diabetes. Since this patient has difficulties achieving ultrafiltration, protection of the cardiovascular system is mandatory, although in CKD we do not have much evidence of the efficacy of the cardioprotective drugs in this situation. This field is extremely important to be studied to reduce patient mortality.

Dr. Pecoits-Filho: Just a comment, it is important to point out that studies and clinical guidelines focusing on the treatment of diabetes in $\mathrm{PD}$ are lacking, pointing to the need of a real effort from the nephrological community to develop this information. We do not even know targets for glucose and $\mathrm{HbA1C}$, how to correct for the constant glucose absorption, and which drug should be utilized. All these questions need to be approached for us to be able to translate them into an improvement in patient outcome.

Inacio Villanueva, Nephrologist, Fundación Leonor Goeckel, Bogota: I have a few comments. First, one thing is to develop guidelines; another is to be able to generalize the recommendations throughout Latin America. However, Brazil and Colombia share similarities, such as the large population with low economic and educational level, usually considered not suitable for PD, mainly due to the lack of studies in the area. But in fact, some patients that live far from large cities and cannot be transferred to a city that has a dialysis center may die without treatment. We cannot follow the guidelines so strictly in Latin America.

Second, despite the high costs of dialysis, we need to offer quality of life, and APD represents an important tool. Initially restricted to a particular group of patients, nowadays APD is a possibility to improve quality of life in children, active working people and perhaps to the general PD population. In CAPD, the patient or helper will have to dedicate too much time for the treatment, generating a negative impact on quality of life and potentially in patient compliance.

Dr. Pecoits-Filho: I would like to comment on the APD issue. At the present time, the introduction of new technology, solutions and devices in PD is very difficult due to the high impact of dialysis in general in the public health system. Particularly in cases of innovation that is not supported by clear evidence of benefit that justifies an increase in cost of the treatment, the introduction of these more expensive tools in clinical practice is usually not an easy process. Despite the fact that I believe that APD and new PD solutions will benefit patients universally, the evidence that they reduce important complications and mortality is lacking and needs to be generated. To develop and carry out a large study to prove these points is not an easy task since the $\mathrm{PD}$ population is small and the effect of single interventions may be not so strong in this population with a large number of risk factors.

Dr. Laura Sola, Hospital Maciel, Montevideo, Uruguay: As mentioned earlier, this is a typical patient in Latin America, who arrives for treatment without the adequate pre-dialysis care. This patient was most likely not really familiar with treatment options and not very well educated. In this regard, I was not surprised by the clinical presentation at the admission. What needs to be clarified, since it is a common complication of the catheter in obese patients, is leakage to the abdominal wall. The prescription in such cases should include more hypertonic solutions to achieve enough ultrafiltration to maintain a good fluid status. Also, this patient, due to his lifestyle (bus driver), will need to be switched to a more flexible treatment modality, such as APD.

Aura Cecilia Cardenas, Dialysis Nurse, Bogota, Colombia: I have 6 years of experience with PD and have not seen a patient with such an early ultrafiltration failure. 
Normally, in such early cases of low drainage volume, there are catheter malfunction or intestinal problems. Usually, patients with ultrafiltration problems do not respond well to $1.5 \%$ glucose solutions. Although this pa- tient had an ultrafiltration of $400 \mathrm{ml}$, this is not a good indicator of peritoneal transport status. The patient should be carefully followed since low compliance to dialysis prescription is very common.

\section{References}

-1 Cusumano AM, Di Gioia C, Hermida O, Lavorato C: The Latin American Dialysis and Renal Transplantation Registry Annual Report 2002. Kidney Int Suppl 2005:S46S52.

-2 Pecoits-Filho R, Abensur H, Cueto-Manzano AM, Dominguez J, Divino Filho JC, Fernandez-Cean J, Ortiz AM, Moretta G, Ramos A, Sanabria M, Sesso R, Paniagua R: Overview of peritoneal dialysis in Latin America. Perit Dial Int 2007;27:316-321.

-3 Korevaar JC, Feith GW, Dekker FW, van Manen JG, Boeschoten EW, Bossuyt PM, Krediet RT: Effect of starting with hemodialysis compared with peritoneal dialysis in patients new on dialysis treatment: a randomized controlled trial. Kidney Int 2003; 64:2222-2228.

-4 Van Biesen W, Vanholder R, Debacquer D, De Backer G, Lameire N: Comparison of survival on CAPD and haemodialysis: statistical pitfalls. Nephrol Dial Transplant 2000;15: 307-311.
5 Sanabria M, Munoz J, Trillos C, Hernandez G, Latorre C, Diaz CS, Murad S, Rodriguez K, Rivera A, Amador A, Ardila F, Caicedo A Camargo D, Diaz A, Gonzalez J, Leguizamon H, Lopera P, Marin L, Nieto I, Vargas E: Dialysis outcomes in Colombia (DOC) study: A comparison of patient survival on peritoneal dialysis vs. hemodialysis in Colombia. Kidney Int Suppl 2008:S165-172.

6 Abbott KC, Glanton CW, Trespalacios FC, Oliver DK, Ortiz MI, Agodoa LY, Cruess DF Kimmel PL: Body mass index, dialysis modality, and survival: analysis of the United States Renal Data System Dialysis Morbidity and Mortality Wave II Study. Kidney Int 2004;65:597-605.

7 Pecoits-Filho R, Campos C, Cerdas-Calderon $\mathrm{M}$, Fortes $\mathrm{P}$, Jarpa $\mathrm{C}$, Just $\mathrm{P}$, Luconi $\mathrm{P}$, Lugon JR, Pacheco A, Paniagua R, Rodriguez K, Sanabria M, Sciaraffia V, Velasco C, De Arteaga J: Policies and health care financing issues for dialysis in Latin America: extracts from the roundtable discussion on the economics of dialysis and chronic kidney disease. Perit Dial Int 2009;29(Suppl 2):S222S226.

8 Bastos K, et al: Socioeconomic status does not impact on peritoneal dialysis clinical outcome: results from the BRAZPD. (abstract). World Congress of Nephrology, Milan, 2009.
9 La Milia V, Limardo M, Cavalli A, Crepaldi M, Locatelli F: Transport of peritoneal membrane assessed before and after the start of peritoneal dialysis. Nephrol Dial Transplant 2009;24:2894-2898.

10 Mushahar L, Lambie M, Tan K, John B, Davies SJ: Long-term changes in solute and water transport. Contrib Nephrol 2009;163:1521.

11 van Olden RW, Guchelaar HJ, Struijk DG, Krediet RT, Arisz L: Acute effects of highdose furosemide on residual renal function in CAPD patients. Perit Dial Int 2003;23: 339-347.

12 Chung SH, Heimburger O, Lindholm B: Poor outcomes for fast transporters on PD: the rise and fall of a clinical concern. Semin Dial 2008;21:7-10.

13 Fortes PC, de Moraes TP, Mendes JG, Stinghen AE, Ribeiro SC, Pecoits-Filho R: Insulin resistance and glucose homeostasis in peritoneal dialysis. Perit Dial Int 2009;29(Suppl 2):S145-S148

14 Foley RN, Parfrey PS, Harnett JD, Kent GM, Murray DC, Barre PE: The impact of anemia on cardiomyopathy, morbidity, and mortality in end-stage renal disease. Am J Kidney Dis 1996;28:53-61. 